\title{
Withholding treatment: What, Whom and Why?
}

When is it permissible for medical professionals to withhold a medical intervention? Several considerations might immediately be deemed morally relevant to such a decision. Does the medical professional believe that the intervention is in the patient's best interests? Is the patient competent, and has she refused to consent to the intervention? These considerations are routinely invoked in end-of-life decision-making. Indeed, in this issue, White et al. present important empirical data concerning the role that law and personal ethical principles play in healthcare professionals' decisions to withhold and withdraw life-sustaining treatment from adults who lack capacity. Other articles in this issue go beyond the end of life context to address provocative questions about the permissibility of (and the rationales for) withholding different kinds of medical interventions from different kinds of (potential) patients.

\section{WHAT IS WITHHELD, AND WHY?}

In the clinical ethics section of this issue, papers by Paddy McQueen and Heidi Metres address the question of whether physicians may justifiably refuse voluntary sterilisation requests of competent women. Both authors acknowledge that such refusals may be grounded by the physician's prediction of future regret amongst putative recipients of the intervention. In response, McQueen argues that anticipated future regret is not a good reason for withholding sterilization, since recognising an individual as an autonomous agent requires that we permit them to make locally justified decisions that they might later regret. On a different approach to the same issue, Metres argues that there is an empirically unjustified discrepancy in the degree of consideration given to potential future regret in decisions to honour requests for sterilisation on the one hand, and decisions to honour requests for fertility treatment on the other.

Also in this section, Patrick Sullivan defends the view that healthcare professionals in mental health units should allow patients to carry out acts of selfharm. This can be construed as enjoining healthcare professionals to withhold a certain kind of medical care in this context. Sullivan raises several arguments, grounded by considerations of autonomy and (perhaps surprisingly) all things considered harm prevention, in favour of an approach that prioritises harm minimisation of self-harm over preventative strategies. Pickard and Pearce's response stresses the importance of distinguishing between secure and non-secure inpatient settings in discussing the harm minimisation approach, and counters that the potential benefits of this approach may be outweighed by potential costs to staff, other patients, and indeed the patient herself.

\section{FROM WHOM IS IT WITHHELD AND WHY?}

These papers raise important questions regarding the permissibility of withholding certain kinds of medical interventions. In some cases though, ethical questions about withholding treatment pertain most saliently to the issue of from whom we are withholding medical treatment.

Triage systems are decision protocols for allocating scarce medical resources in scenarios where need outstrips available resources; since all cannot be treated, then we need principles to determine who of the many in need should be treated. In their contribution this issue, Azgad Gold and Rael D Strous outline how the conventional view of triage may be understood to maintain a broadly prioritarian approach to this issue. The conventional view holds that, amongst individuals who have some threshold non-remote chance of survival, medical treatment should be allocated in accordance with the urgency of each patient's need, regardless of other contextual factors. The authors go on to argue that this impersonal, universalist 'worst-first' conventional approach is inappropriate in cases in which we must weigh the (potentially urgent) medical needs of a known perpetrator of a deliberate violent crime (such as a terrorist attack), and the (potentially less urgent) medical needs of the victims of their attack. Here, Gold and Strous argue that the conventional view overlooks a number of morally relevant features. Whilst acknowledging that, ceteris paribus, basic humanistic concern enjoins medics to provide treatment even to perpetrators of violent crimes, the authors deploy considerations from retributive, distributive, and collective justice, to argue in favour of a qualified 'victim-first' approach to triage in these cases. In doing so, they suggest that the importance of causal responsibility on their justiceoriented strategy means that the 'victimfirst' approach should be reserved for only deliberate and extreme violent crimes by known perpetrators.

In his commentary on this paper, Michael Ardagh raises the concern that this victim-first approach is not translatable, due to the significant potential for error in assessments of worthiness for treatment. Such errors may also lead to errors in patient-management. Mark Wicclair echoes Ardagh's epistemic concern and also identifies some putative argumentative gaps in Gold and Strous' three-pronged justice-oriented strategy. Notwithstanding these putative gaps, Wicclair raises a more general concern by questioning whether it is appropriate for physicians to act as agents of justice.

Gold and Strous attend to these criticisms in a response piece, and their reply to the epistemic concern is particularly notable. In addition to stressing the value in considering the terror-triage dilemma as a thought experiment, they also provide the real life case of the 2009 Fort Hood shooting as one in which their approach seems to be directly translatable. This is a convincing example of a case in which there can be little doubt about the identity of the perpetrator of a deliberate violent act. However, there is room for further discussion about whether Gold and Strous' justice-oriented argument might require a significant degree of credence about the perpetrator's moral (as well as causal) responsibility for their violent actions, if their victim-first approach is to be truly grounded by justice-oriented considerations. Naturally though, this would take the discussion into much deeper epistemic, and perhaps even metaphysical waters, which are arguably best avoided in discussions of such pressing practical concern. 